\title{
Surveillance des substances contrôlées en établissements de santé : une contribution à la gestion de la crise des opioïdes au Canada
}

\author{
par Manon Videau, Maxime Thibault, Denis Lebel, Suzanne Atkinson et Jean-François Bussières
}

\begin{abstract}
RÉSUMÉ
Contexte : La consommation des substances contrôlées et plus particulièrement des opioïdes est un enjeu de santé publique. Le Canada se situe au deuxième rang des plus gros consommateurs d'opioïdes dans le monde. L'utilisation de ces substances est associée à des problèmes de mésusage. À preuve, une crise des opioïdes sévit en Amérique du Nord.

Objectifs : Décrire et analyser les tendances de consommation des substances contrôlées au sein d'un établissement de santé de 2003-2004 à 2017-2018. Proposer un outil de surveillance de la consommation des substances contrôlées dans un établissement de santé.
\end{abstract}

Methodologie : Étude descriptive rétrospective. À partir du logiciel de gestion des approvisionnements, nous avons extrait les données de consommation de toutes les substances contrôlées du $1^{\text {er }}$ avril 2003 au 31 mars 2018. Les données ont été exprimées selon l'index de la classification Anatomical Therapeutic Chemical en nombre de doses définies journalières (DDJ) pour 1000 jours-présence avec les valeurs de DDJ proposées par l'Organisation mondiale de la santé. Seules des statistiques descriptives ont été effectuées.

Resultats : Durant les 15 dernières années, la consommation des substances contrôlées a diminué de $43 \%$ au sein de notre établissement (min. : 739; max. : 1292 DDJ/1000 jours-présence par année). De 20032004 à 2017-2018, les principales classes thérapeutiques consommées par ordre décroissant étaient : opioïdes, hypnotiques et sédatifs, anxiolytiques et anesthésiques généraux. Les principales molécules opioïdes consommées en 2017-2018 sont l'hydromorphone et la morphine injectable.

Conclusions : Cette étude descriptive rétrospective montre une diminution de la consommation des substances contrôlées au sein de notre établissement de 2003-2004 à 2017-2018. Elle démontre la faisabilité de développer un outil de surveillance de la consommation des substances contrôlées en établissement de santé. Une telle approche pourrait être implantée à large échelle afin de favoriser les comparaisons entre les établissements.

Mots clés : consommation, substances contrôlées, substances désignées, opioïdes, dose définie journalière, gouvernance des substances contrôlées

\begin{abstract}
Background: The use of controlled substances, especially opioids, is a public health concern. Canada is the country with the second greatest opioid use in the world. The use of these substances is associated with problems of misuse, as evidenced by North America's opioid crisis.

Objectives: To describe and analyze usage patterns for controlled substances in a health care facility from $2003 / 04$ to $2017 / 18$, and to propose a tool for monitoring the use of controlled substances in this setting.
\end{abstract}

Method: In this retrospective descriptive study, usage data for all controlled substances were extracted from the institution's supply management software for the period April 1, 2003, to March 31, 2018. The data are presented according to the Anatomical Therapeutic Chemical classification in terms of number of Defined Daily Doses (DDD) per 1000 inpatient-days, using the DDD values proposed by the World Health Organization. Only descriptive statistics were determined.

Results: During the last 15 years, use of controlled substances at the study facility dropped by $43 \%$ (min. 739 and max. 1292 DDD/1000 inpatient-days per year). From 2003/04 to 2017/18, the main therapeutic classes consumed (in decreasing order) were opioids, hypnotics and sedatives, anxiolytics, and general anesthetics. The main opioid molecules consumed in 2017/18 were hydromorphone and injectable morphine.

Conclusions: This retrospective descriptive study showed a decrease in the consumption of controlled substances in the study facility from $2003 / 04$ to $2017 / 18$. It also demonstrated the feasibility of developing a tool for monitoring the use of controlled substances in a health care facility. This approach could be implemented at a larger scale to foster comparisons between facilities.

Keywords: consumption, controlled substances, designated substances, opioids, defined daily dose, controlled substances stewardship

J. Can. Pharm. Hosp. 2020;73(2):116-24 


\section{INTRODUCTION}

A Canada, on entend par substances contrôlées (aussi appelées substances désignées), toute substance inscrite à l'une ou l'autre des annexes I, II, III, IV ou VI de la Loi règlementant certaines drogues et autres substances (LRCDAS) ${ }^{1}$. Cette loi concerne les stupéfiants, les drogues contrôlées, les substances ciblées, les benzodiazépines et les précurseurs. Ces drogues sont divisées en catégories basées sur le potentiel d'abus ou d'accoutumance. Les substances contrôlées comprennent autant les drogues illicites en vente libre que les médicaments prescrits $^{2}$.

Utilisés depuis plusieurs décennies pour le traitement des douleurs aigües, postopératoires et palliatives, les opioïdes ont vu leur usage s'élargir avec la prise en charge de la douleur chronique non cancéreuse. Ainsi, on observe depuis les années 2000 une augmentation du nombre de prescriptions d'opioïdes aux ÉtatsUnis ${ }^{3}$. Parallèlement, 70237 décès par surdose ont été dénombrés aux États-Unis en 2017, dont les deux tiers étaient liés aux opiö̈des ${ }^{4}$. Pour la même année, on a dénombré 4034 décès apparemment liés aux opioïdes au Canada 5 . Selon l'Organisme international de contrôle des stupéfiants, le Canada et les ÉtatsUnis sont les deux principaux pays consommateurs d'opioïdes sur ordonnance dans le monde ${ }^{6}$. Ainsi, l'augmentation croissante de l'utilisation d'opioïdes, avec ou sans ordonnance, est associée à une augmentation du nombre de décès par surdosage dans le contexte de ce qu'on appelle désormais la " crise des opioïdes » Cette crise constitue un problème de santé publique majeur en Amérique du Nord.

Divers organismes ont mis en place des programmes pour sensibiliser les professionnels de la santé et les patients aux risques liés à l'utilisation des substances contrôlées. Aux États-Unis, la Joint Commission dispose de critères précis pour juger des mesures d'évaluation de la douleur mises en place et de la prescription des opioïdes dans les hôpitaux américains et elle encourage la mise en place de programmes de gestion des opioïdes (opioid stewardship) $)^{8-10}$.

Au Canada, ISMP Canada (Institute for Safe Medication Practices Canada) soutient un programme semblable, qui se définit comme un ensemble d'interventions coordonnées pour améliorer, monitorer et évaluer l'utilisation des opiö̈des dans le but de supporter et de protéger la vie humaine (traduction libre $)^{11}$.

Les prescripteurs qui exercent dans les hôpitaux sont à l'origine de la mise en œuvre de nombreux traitements aux opiö̈des, tant pour les soins aigus (p. ex. après une opération chirurgicale) que pour les soins chroniques (p. ex. douleurs chroniques réévaluées après l'hospitalisation, congé donné en soins palliatifs à domicile). Les substances contrôlées inscrites à la liste locale d'un établissement, les protocoles et feuilles d'ordonnances prérédigées mises en place ont un impact considérable sur la pratique, le choix et les quantités des substances contrôlées prescrites.
En outre, on reconnaît les risques d'abus et de mésusage par les professionnels de la santé ${ }^{12}$. En effet, l'accès des professionnels de la santé aux substances contrôlées dans le cadre de leur pratique facilite le détournement potentiel de ces substances pour une utilisation personnelle ou même du trafic. Ce risque de détournement de substances contrôlées peut influencer l'utilisation et il représente également un risque de sécurité pour les patients, étant donné que des doses de ces médicaments peuvent être enregistrées au nom des patients sans leur être réellement administrées ${ }^{13}$. Ainsi les établissements de santé sont confrontés à d'importants enjeux en termes de respect des exigences règlementaires, d'amélioration continue de la qualité des soins et de prévention du détournement des substances contrôlées. C'est dans ce contexte que la Société canadienne des pharmaciens d'hôpitaux a récemment publié des recommandations entourant la gestion et la prévention du détournement des substances contrôlées en établissement de santé ${ }^{14}$.

L'objectif principal de cette étude vise à décrire et à analyser les tendances de consommation des substances contrôlées au sein d'un établissement de santé de 2003-2004 à 2017-2018. L'objectif secondaire consiste à proposer un outil de surveillance de la consommation des substances contrôlées dans un établissement de santé, qui devrait être jumelé à des mesures d'encadrement des prescriptions et de formation données aux prescripteurs et aux usagers.

\section{MÉTHODES}

\section{Conception de l'étude}

Il s'agit d'une étude descriptive rétrospective se déroulant au CHU Sainte-Justine, un centre hospitalier universitaire mèreenfant de 500 lits situé à Montréal, Québec, Canada. L'établissement offre l'ensemble des soins généraux et spécialisés groupés dans les disciplines suivantes : médecine (y compris médecine interne et hémato-oncologie), chirurgie, gynéco-obstétrique, soins intensifs, psychiatrie, néonatologie et pouponnière, urgence.

\section{Origine des données}

Les données de consommation des substances contrôlées ont été extraites par année financière entre le $1^{\text {er }}$ avril 2003 et le 31 mars 2018 à partir du logiciel de gestion des approvisionnements (GRM Espresso $^{\mathrm{MD}}$, Logibec, Montréal, Canada). Une année financière débute le $1^{\mathrm{er}}$ avril d'une année et se termine le 31 mars de l'année suivante. Ces données correspondent aux sorties unitaires annuelles réalisées sur le logiciel de gestion des stocks de la pharmacie. Le nombre d'admissions et de jours-présence par année financière a également été recueilli pour décrire les volumes d'activités d'hospitalisation de courte durée.

Les substances contrôlées ont été déterminées à partir du cadre législatif en vigueur ${ }^{15-17}$. Les substances marquées de la lettre « $\mathrm{N}$ » correspondent aux stupéfiants, médicaments régis par le 
règlement sur les stupéfiants (p. ex. morphine, codéine, oxycodone, hydromorphone); les substances marquées de la lettre " $\mathrm{C}$ » correspondent aux drogues contrôlées, médicaments régis par le règlement sur les aliments et les drogues (p. ex. amphétamine, méthylphenidate, phénobarbital); les substances marquées des lettres « $\mathrm{T} / \mathrm{C}$ » correspondent aux médicaments régis par le Règlement sur les benzodiazépines et autres substances ciblées (p. ex. alprazolam, diazépam) ${ }^{15-17}$.

\section{Analyses}

Les données de chaque substance contrôlée sont exprimées selon l'index de la classification anatomique, thérapeutique et chimique (ATC) et la formulation des médicaments. Les quantités dispensées sont exprimées en milligrammes par année et en nombre de doses définies journalières (DDJ) pour 1000 joursprésence par année. Les données sont également exprimées en équivalent en milligrammes de morphine (EMM) orale pour 1000 jours-présence par année pour la classe des opioïdes (N02A), des alcaloïdes de l'opium et dérivés (R05D) et des anesthésiques généraux (N01A); enfin, les données sont exprimées en équivalent en milligrammes de diazépam (EMD) oral pour 1000 joursprésence par année pour les classes des anxiolytiques (N05B), des hypnotiques et des sédatifs (N05C).

\section{Dose définie journalière (DDJ)}

La dose définie journalière correspond à la dose d'entretien moyenne par jour pour l'indication principale d'un médicament pour l'adulte $(70 \mathrm{~kg})$. Définie par l'Organisation mondiale de la santé (OMS), cette norme de mesure permet de convertir les quantités physiques des médicaments (p. ex. comprimés, fioles, inhalateurs) en une unité de mesure standardisée et permet ainsi d'évaluer les tendances d'utilisation des médicaments.

Lorsque cela était possible, les investigateurs ont utilisé les valeurs de DDJ proposées par l'OMS (tableau 1) 14,15,18,19. Les DDJ des associations de produits ont été considérées comme la DDJ de la substance principale, selon les lignes directrices de l'OMS. Le calcul des données s'effectue comme suit : nombre total de milligrammes consommés par année / ratio DDJ en mg divisé par le nombre total de jours-présence $\times 1000$.

Toutefois, l'OMS n'a pas établi de DDJ pour les formes topiques et pour les anesthésiques généraux et locaux. Compte tenu de la consommation marginale de cocaïne ophtalmique et topique, d'hydroxyamphétamine ophtalmique et de nabilone, nous avons exclu ces médicaments de l'analyse. Ainsi, nous nous sommes basés sur les règles générales utilisées par l'OMS pour établir une DDJ par médicament des produits suivants : kétamine, rémifentanil, sufentanil, etomidate et hydroxyamphetamine ${ }^{20,21}$.

\section{Tableau 1 (partie 1 de 2). DDJ et ratio d'équivalent en milligrammes de morphine ou de diazépam utilisés pour les calculs}

\begin{tabular}{|c|c|c|c|}
\hline $\begin{array}{l}\text { Médicament et voie } \\
\text { d'administration* }\end{array}$ & $\begin{array}{l}1 \mathrm{DDJ} \\
(\mathrm{mg})\end{array}$ & $\begin{array}{l}\text { Équivalent en } \\
\text { milligrammes de } \\
\text { morphine oralet }\end{array}$ & $\begin{array}{l}\text { Équivalent en } \\
\text { milligrammes de } \\
\text { diazépam oralf }\end{array}$ \\
\hline \multicolumn{4}{|l|}{ Opioïdes (N02A) } \\
\hline Buprenorphine INJ & 1,2 & 75 & NA \\
\hline Codeine INJ & 240 & 0,3 & $N A^{\prime}$ \\
\hline Codeine PO & 100 & 0,1 & NA \\
\hline Fentanyl INJ & 0,6 & 200 & NA \\
\hline Fentanyl PO & 0,6 & 100 & NA \\
\hline Fentanyl TOPI & 1,2 & 100 & NA \\
\hline Hydromorphone INJ & 4 & 17,5 & NA \\
\hline Hydromorphone PO & 20 & 5 & NA \\
\hline Hydromorphone $\mathrm{R}$ & 4 & 5 & NA \\
\hline Methadone INJ & 25 & 13,5 & NA \\
\hline Methadone PO & 25 & 4,7 & NA \\
\hline Morphine INJ & 30 & 3 & NA \\
\hline Morphine PO & 100 & 1 & NA \\
\hline Morphine R & 30 & 1,5 & NA \\
\hline Nalbuphine INJ & 80 & 3 & NA \\
\hline Oxycodone PO & 75 & 1,5 & NA \\
\hline Tramadol PO & 300 & 0,2 & NA \\
\hline \multicolumn{4}{|c|}{ Alcaloïdes de l'opium et dérivés (R05D) } \\
\hline Normethadone PO & 25 & 4,7 & NA \\
\hline \multicolumn{4}{|c|}{ Antagonistes des récepteurs opioïdes périphériques (A06A) } \\
\hline Methylnaltrexone INJ & 6 & NA & NA \\
\hline \multicolumn{4}{|c|}{ Anesthésiques généraux (N01A) } \\
\hline Etomidate INJ & 21 & NA & NA \\
\hline Ketamine INJ & 350 & NA & NA \\
\hline Ketamine PO & 120 & NA & NA \\
\hline Remifentanil INJ & 0.6 & 100 & NA \\
\hline Sufentanil INJ & 0.06 & 2000 & NA \\
\hline
\end{tabular}


Tableau 1 (partie 2 de 2). DDJ et ratio d'équivalent en milligrammes de morphine ou de diazépam utilisés pour les calculs

\begin{tabular}{|c|c|c|c|}
\hline $\begin{array}{l}\text { Médicament et voie } \\
\text { d'administration* }\end{array}$ & $\begin{array}{l}1 \text { DDJ } \\
\text { (mg) }\end{array}$ & $\begin{array}{l}\text { Équivalent en } \\
\text { milligrammes de } \\
\text { morphine oralet }\end{array}$ & $\begin{array}{l}\text { Équivalent en } \\
\text { milligrammes de } \\
\text { diazépam oralł }\end{array}$ \\
\hline \multicolumn{4}{|l|}{ Anxiolytiques (N05B) } \\
\hline Alprazolam PO & 1 & NA & 2 \\
\hline Clobazam PO & 20 & NA & 1 \\
\hline Diazepam INJ & 10 & NA & 1 \\
\hline Diazepam PO & 10 & NA & 1 \\
\hline Diazepam R & 10 & NA & 1 \\
\hline Lorazepam INJ & 2,5 & NA & 2.5 \\
\hline Lorazepam PO & 2,5 & NA & 2.5 \\
\hline Oxazepam PO & 50 & NA & 2.5 \\
\hline \multicolumn{4}{|c|}{ Hypnotiques et sédatifs (N05C) } \\
\hline Amobarbital INJ & 100 & NA & NA \\
\hline Chloral PO & 1000 & NA & NA \\
\hline Chloral R & 1000 & NA & NA \\
\hline Midazolam INJ & 15 & NA & 3 \\
\hline Midazolam PO & 15 & NA & 3 \\
\hline Nitrazepam PO & 5 & NA & 0.5 \\
\hline Pentobarbital INJ & 100 & NA & NA \\
\hline Pentobarbital PO & 100 & NA & NA \\
\hline Phenobarbital PO & 100 & NA & NA \\
\hline Secobarbital PO & 100 & NA & NA \\
\hline Temazepam PO & 20 & NA & 1 \\
\hline Zolpidem PO & 10 & NA & 0.5 \\
\hline \multicolumn{4}{|l|}{ Psychostimulants (N06B) } \\
\hline Amphetamine PO & 15 & NA & NA \\
\hline Dexamphetamine PO & 15 & NA & NA \\
\hline Hydroxyamphetamine PO & 15 & NA & NA \\
\hline Lisdexamphetamine INJ & 30 & NA & NA \\
\hline Methylphenidate PO & 30 & NA & NA \\
\hline \multicolumn{4}{|l|}{ Androgènes (G03B) } \\
\hline Methyltestosterone PO & 25 & NA & NA \\
\hline Testosterone INJ & 18 & NA & NA \\
\hline Testosterone PO & 120 & NA & NA \\
\hline Testosterone TOPI & 50 & NA & NA \\
\hline
\end{tabular}

L'utilisation des DDJ permet la présentation et la comparaison statistique de la consommation de médicaments, mais elle ne reflète pas l'exposition réelle de patients aux substances contrôlées. En effet, il existe une importante différence entre la dose utilisée en pratique clinique de beaucoup d'opioïdes et les DDJ de l'OMS ${ }^{22}$. L'interprétation des études d'utilisation d'opioïdes par la population reposant sur les DDJ doit se faire avec précaution ${ }^{22}$. Ainsi, en complément des DDJ, on recommande l'analyse en équivalent en milligrammes de morphine orale, car la morphine est le médicament de référence pour le traitement de la douleur ${ }^{18,23-25}$. Il en est de même pour les benzodiazépines, étant donné que les DDJ reflètent mal l'exposition clinique, car elles ne prennent pas en compte la puissance de ces molécules. Ainsi, les investigateurs ont réalisé une analyse en équivalent en milligrammes de diazépam en complément des DDJ ${ }^{25}$. Cette unité permet une estimation de l'exposition pharmacologique de la population et une meilleure estimation de la puissance sédative ${ }^{25}$.

\section{Équivalent en milligrammes de morphine orale (EMM)}

À partir des données de la littérature, des ratios d'équivalent en milligrammes de morphine orale par principe actif et par voie d'administration ont été établis pour les molécules de la classe des opioïdes (N02A), des alcaloïdes de l'opium et dérivés (R05D) et des anesthésiques généraux (N01A) (tableau 1) ${ }^{18,20-25}$. Les ratios ont par la suite été approuvés par un anesthésiste et un pharmacien. Les données ont été calculées comme suit : nombre total de DDJ du médicament considéré $\times$ le ratio d'équivalent de morphine $\times$ le ratio DDJ en $\mathrm{mg}$ divisé par le nombre total de jours-présence $\times 1000$. 


\section{Équivalent en milligrammes de diazépam (EMD)}

À partir du tableau d'équivalence des benzodiazépines proposé par Ashton ${ }^{19}$ et des données de la littérature, des ratios d'équivalent en milligrammes de diazépam ont été déterminés pour les substances de la classe des anxiolytiques (N05B) et des hypnotiques et sédatifs (N05C) (tableau 1). Les données ont été calculées comme suit : DDJ/1000 jours-présence de la molécule $\times$ le nombre de milligrammes d'équivalent de diazépam.

\section{RÉSULTATS}

De 2003-2004 à 2017-2018, la consommation globale des substances contrôlées a diminué de $43 \%$ au sein de notre établissement (min. 739 DDJ par 1000 jours-présence en 20172018; max. 1292 DDJ par 1000 jours-présence en 2003-2004).

Au cours de cette période, les principales classes thérapeutiques consommées sont par ordre décroissant : la classe des opioïdes (N02A), les hypnotiques et sédatifs (N05C), les anxiolytiques (N05B) et les anesthésiques généraux (N01A) (figure 1).

\section{Les opioïdes}

De 2003-2004 à 2017-2018, la consommation des opioïdes a diminué de $46 \%$ avec un maximum en 2012-2013 (610 DDJ par 1000 jours-présence) et un minimum en 2017-2018 (314 DDJ par 1000 jours-présence).

En 2017-2018, les principales molécules consommées sont l'hydromorphone injectable (100 DDJ par 1000 jours-présence), suivie par la morphine injectable (70 DDJ par 1000 joursprésence), la codéine orale (58 DDJ par 1000 jours-présence) et le fentanyl injectable (45 DDJ par 1000 jours-présence) (figure $2 \mathrm{~A})$. Des résultats similaires sont observés en équivalent en milligrammes de morphine $\left(R^{2}=0,98\right)$. Ainsi, l'hydromorphone injectable (7029 EMM par 1000 jours-présence) est la principale molécule consommée, suivie de la morphine injectable (6307 EMM par 1000 jours-présence) et du fentanyl injectable (5454 EMM par 1000 jours-présence). Toutefois, la codéine, dont la puissance est plus faible que celle de la morphine, se retrouve loin derrière la morphine injectable et le fentanyl injectable (583 EMM par 1000 jours-présence) (figure 3A).

\section{Les hypnotiques et sédatifs}

De 2003-2004 à 2017-2018, la consommation des hypnotiques et des sédatifs a diminué de $76 \%$ avec un maximum en 2005-2006 (283 DDJ par 1000 jours-présence) et un minimum en 2017-2018 (65 DDJ par 1000 jours-présence). En 2017-2018, la principale molécule consommée est le midazolam injectable (58 DDJ par 1000 jours-présence). La principale modification observée est l'abandon du nitrazépam oral en 2015-2016 (figure 2B). Des résultats similaires sont observés en équivalent en milligrammes de diazépam $\left(R^{2}=0,91\right)$ (figure $3 \mathrm{~B}$ ). On note 174 EMD par 1000 jours-présence pour le midazolam injectable.

\section{Les anxiolytiques}

De 2003-2004 à 2017-2018, la consommation des anxiolytiques a diminué de $24 \%$ avec un maximum de $292 \mathrm{DDJ}$ par 1000 jours-présence en 2007-2008 et un minimum de 184 DDJ par 1000 jours-présence en 2015-2016. Les principales variations observées sont la diminution de la consommation de lorazépam injectable depuis 2013 et l'augmentation de clobazam oral depuis 2014 (figure 2C). Des données similaires sont retrouvées en $\operatorname{EMD}\left(R^{2}=0,96\right)$ (figure 3C).

\section{Les anesthésiques généraux}

De 2003-2004 à 2017-2018, la consommation des anesthésiques généraux a augmenté de $7 \%$, avec un maximum

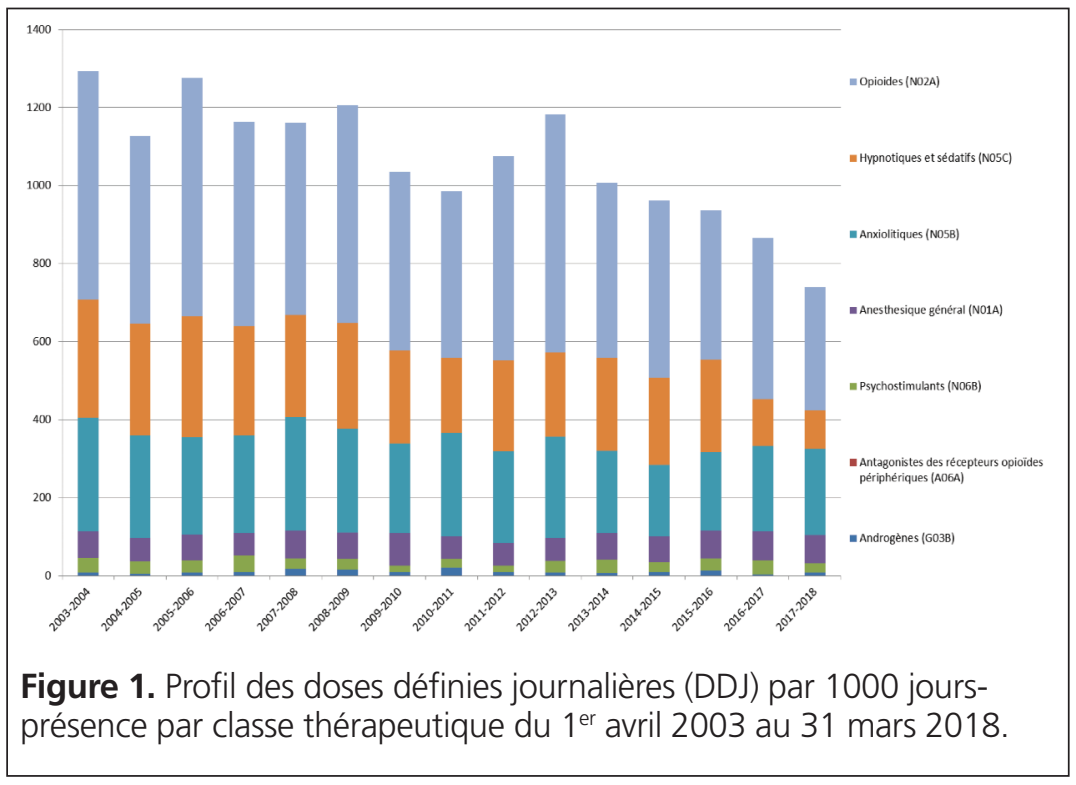



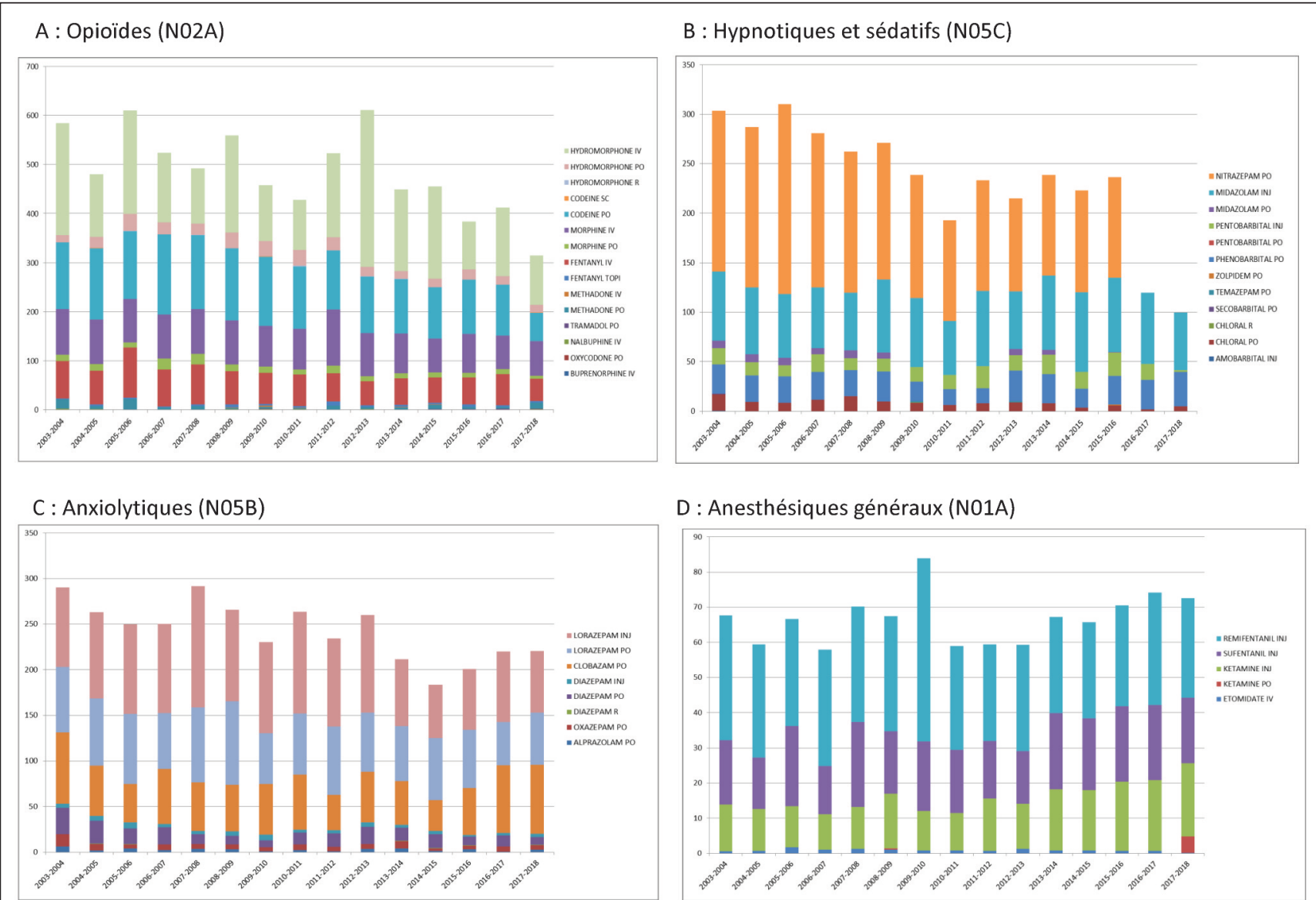

Figure 2. Profils de consommation en doses définies journalières (DDJ) par 1000 jours-présence, du $1^{\text {er }}$ avril 2003 au 31 mars 2018, pour (A) la classe des opioïdes, N02A; (B) des hypnotiques et sédatifs, N05C; (C) des anxiolytiques, N05B; et (D) des anesthésiques généraux, N01A. INJ = injectable, PO = per os, $\mathrm{R}=$ rectal, SC = sous-cutané.

de 84 DDJ par 1000 jours-présence en 2009-2010 et un minimum de 58 DDJ par 1000 jours-présence en 2006-2007. Les principales molécules consommées en 2017-2018 sont le remifentanil injectable (28 DDJ par 1000 jours-présence), la kétamine injectable (21 DDJ par 1000 jours-présence) et le sufentanil injectable (19 DDJ par 1000 jours-présence) (figure 2D). Des données similaires sont retrouvées en $\operatorname{EMM}\left(R^{2}=0,86\right)$ (figure 3D).

\section{Autres}

En ce qui concerne les psychostimulants, la principale molécule consommée en 2017-2018 est le méthylphenidate oral (23 DDJ par 1000 jours-présence) (annexe 1, disponible au https://www.cjhp-online.ca/index.php/cjhp/issue/view/ 196/showToc). La testostérone injectable est le principal androgène consommé en 2017-2018 (annexe 2, disponible au https://www.cjhp-online.ca/index.php/cjhp/issue/view/ 196/showToc).

\section{DISCUSSION}

Cette étude originale présente l'évolution de la consommation des substances contrôlées sur une période de 15 ans au sein d'un établissement hospitalier mère-enfant canadien. Des travaux similaires sur les benzodiazépines ont notamment été effectués en Australie ${ }^{26}$.

Notre étude propose une approche similaire à celle utilisée dans la gestion des antimicrobiens ${ }^{27}$. Afin de réduire les risques de résistance associés à la surutilisation des antimicrobiens, plusieurs autorités conseillent de surveiller le nombre de DDJ et le nombre de jours de traitement aux antimicrobiens afin de déterminer des changements de pratique qui ne respectent pas les règles d'utilisation ${ }^{28-30}$. Cette approche est associée à un meilleur contrôle d'utilisation en permettant une analyse des tendances ${ }^{31}$. Dans le cas des antimicrobiens, les établissements de santé ont développé des outils facilitant l'extraction des données et la production de rapports synthèses, ce qui n'est pas forcément le cas pour d'autres classes thérapeutiques de médicaments, comme les substances contrôlées. 

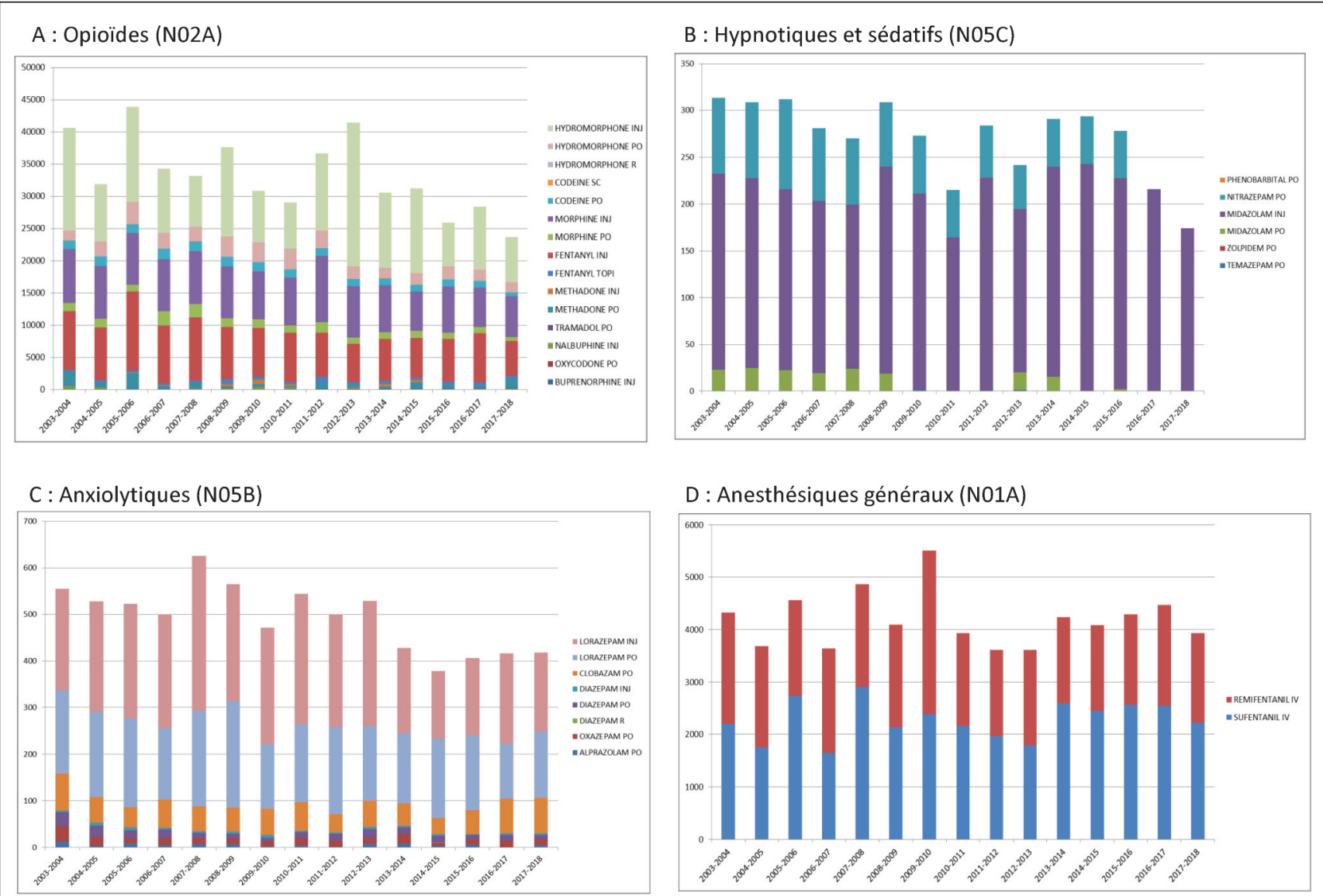

Figure 3. Profils de consommation, du 1er avril 2003 au 31 mars 2018, en équivalent en milligrammes de morphine par 1000 jours-présence pour (A) la classe des opioïdes, N02A, et (D) des anesthésiques généraux, N01A; et en équivalent en milligrammes de diazepam par 1000 jours-présence pour (B) la classe des hypnotiques et sédatifs, N05C, et (C) des anxiolytiques, N05B. INJ = injectable, PO = per OS, R = rectal, SC = sous-cutané.

Nous observons une diminution de $43 \%$ de la consommation globale des substances contrôlées de 2003-2004 à 2017-2018 ainsi qu'une diminution de la plupart des sous-classes évaluées, à l'exception des anesthésiques généraux. Plusieurs facteurs sont associés à cette diminution, notamment le virage ambulatoire en chirurgie, qui déplace de l'hôpital au domicile la prise de certaines doses par le patient, le recours à de nouvelles techniques chirurgicales moins invasives (p. ex. laparoscopie), la pénurie de certains médicaments (p. ex. opioïdes fabriqués par Sandoz en 2012, lorazépam à quelques reprises), le recours à l'anesthésie sans opioïdes (c.-à-d. kétamine, dexmédétomidine, lidocaine, etc. ${ }^{32}$ ), le retrait de la codéine comme analgésique postopératoire au profit d'anti-inflammatoires non stéroidiens et de morphine ainsi que le retrait de la codéine orale des trousses d'automédication en obstétrique. En 2013, Santé Canada a recommandé que la codéine ne soit administrée qu'aux patients d'âge égal ou supérieur à 12 ans $^{33}$. En 2016, un nouvel avis a été publié afin de préciser les situations cliniques où la codéine ne devrait pas être utilisée ${ }^{34}$. En 2019, un autre avis a été publié, déconseillant l'utilisation de produits contre la toux et le rhume contenant des opioïdes pour les enfants et les adolescents ${ }^{35}$. À l'échelle du Canada, l'Institut canadien d'information sur la santé a noté une diminution de la quantité globale de $4,9 \%$ des opioïdes dispensés entre 2012 et 2016, soit 238 millions de DDJ en 2012 contre 226 millions de DDJ d'opioïdes en $2016^{36}$. Cette réduction nationale et locale n'est pas étrangère à la crise des opioïdes qui sévit au Canada et aux différentes initiatives de sensibilisation et d'actions mises en place au cours de la dernière décennie ${ }^{37}$.

Bien que la diminution de la consommation d'opioïdes semble nécessaire au Canada, elle ne doit pas se faire au détriment de la prise en charge de la douleur des patients. En effet, l'augmentation de la consommation des opioïdes a longtemps été considérée comme un signe de meilleure prise en charge de la douleur (lien avec le PIB des pays et indicateur de développement humain élevé). Il existe donc un équilibre précaire entre une prise en charge optimale de la douleur et les prescriptions excessives, ces dernières pouvant conduire au mésusage, au détournement et à la dépendance ${ }^{38}$. 
En établissement de santé, la gestion des substances contrôlées est généralement bien encadrée compte tenu des exigences législatives et normatives et du circuit du médicament ${ }^{15-17}$. Par exemple, l'acquisition de substances contrôlées requiert une signature électronique de la part d'un pharmacien lors de l'achat et de la confirmation de la réception. L'entreposage à la pharmacie est sécurisé dans une voûte verrouillée sous surveillance avec la présence de caméras de surveillance. La dispensation et l'administration de doses de substances contrôlées aux patients requièrent la signature d'un soignant et d'un témoin et le processus repose sur l'utilisation de registres détaillés pour chaque étape applicable. En outre, des armoires sécurisées et parfois automatisées sont utilisées pour l'entreposage et la dispensation dans les unités de soins et les cliniques externes des établissements de santé. Toutefois, en dépit de toutes ces mesures de gestion, il n'existe pas de bonnes pratiques standardisées déterminant les modalités de surveillance de l'utilisation des substances contrôlées en établissement de santé, y compris les rapports de consommation.

Notre étude met en évidence l'intérêt de développer un programme de gestion des substances contrôlées, sur le même principe que la gestion des antimicrobiens. Par exemple, un sous-comité du comité de pharmacologie pourrait consulter périodiquement le profil de consommation des substances contrôlées en utilisant les DDJ, les EMM et les EMD, s'intéresser aux demandes de modifications de la liste locale, proposer des rétroactions aux grands prescripteurs de ces substances, encourager la tenue d'audits ciblés afin de réévaluer la pertinence de la prescription d'opioïdes. Dans la foulée des pratiques organisationnelles requises et de la norme sur la gestion des médicaments proposées par Agrément Canada, un critère explicite pourrait favoriser la gestion des substances contrôlées.

\section{Limites}

Cette étude comporte des limites. Les données recueillies correspondent à celles de la distribution (c.-à-d. quantités dispensées par la pharmacie au moyen des armoires automatisées ou de la distribution unitaire quotidienne au nom des patients) et incluent toutes les quantités mises au rebut ou détruites (doses partielles, expirées, etc.). L'analyse macro proposée ne permet pas de déterminer les situations avérées de vols ou de chapardage, compte tenu qu'elles sont limitées en occurrences et en quantités. Une analyse des données de consommation réelle par patient, par pathologie ou par parcours de soins pourrait apporter un éclairage complémentaire aux risques de mésusage. Le concept de DDJ a été élaboré dans un contexte de soins aux adultes. En gestion des antimicrobiens, nous utilisons plutôt le concept de nombre de jours de traitement, lequel serait moins pertinent pour décrire l'utilisation des substances contrôlées. À la différence des antimicrobiens, la dose des substances contrôlées varie selon l'indication, l'état clinique, la tolérance et l'exposition préalable.

\section{CONCLUSION}

La consommation des substances contrôlées et plus particulièrement des opioïdes est un enjeu de santé publique. Il y a une diminution de la consommation des substances contrôlées au sein de notre établissement de 2003-2004 à 2017-2018. Cette étude démontre la faisabilité de développer un outil de surveillance de la consommation des substances contrôlées en établissement de santé. Une telle approche pourrait être implantée à large échelle afin de favoriser les comparaisons entre les établissements.

\section{Références}

1. Loi règlementant certaines drogues et autres substances. Publié au : http://lawslois.justice.gc.ca/fra/lois/C-38.8/page-1.html\#h-2. Consulté le 22 mai 2019.

2. Substances contrôlées et précurseurs chimiques. Gouvernement du Canada; 2018. Publié au : https:/www.canada.ca/fr/sante-canada/services/ preoccupations-liees-sante/substances-controlees-precurseurs-chimiques.html. Consulté le 22 mai 2019.

3. Overdose death rates. National Institute on Drug Abuse; 2019. Publié au : https://www.drugabuse.gov/related-topics/trends-statistics/overdose-deathrates. Consulté le 22 mai 2019.

4. Seth P, Scholl L, Rudd RA, Bacon S. Overdose deaths involving opioids, cocaine, and psychostimulants — United States, 2015-2016. MMWR Morb Mortal Wkly Rep. 2018;67:349-58.

5. National report: apparent opioid-related deaths in Canada. Gouvernement du Canada. Publié au : https://infobase.phac-aspc.gc.ca/datalab/nationalsurveillance-opioid-mortality.html. Consulté le 22 mai 2019.

6. International Narcotics Control Board. Opioid consumption motion chart. Board of Regents of the University of Wisconsin System; 2012. Publié au : https://ppsg.medicine.wisc.edu/chart. Consulté le 22 mai 2019.

7. Mesures fédérales concernant les opioüdes. Gouvernement du Canada. Publié au : https://www.canada.ca/fr/sante-canada/services/dependance-aux-drogues/ consommation-problematique-medicaments-ordonnance/opioides/mesuresfederales.html. Consulté le 22 mai 2019.

8. Joint Commission enhances pain assessment and management requirements for accreditated hospitals. Perspectives [Official Newsletter of The Joint Commission]. 2017;37(7):1-3. Publié au: https://www.jointcommission.org/ assets/1/18/Joint_Commission_Enhances_Pain_Assessment_and_ Management_Requirements_for_Accredited_Hospitals1.PDF. Consulté le 22 mai 2019.

9. Weiner SG, Price CN, Atalay AJ, Harry EM, Pabo EA, Patel R, et al. A health system-wide initiative to decrease opioid-related morbidity and mortality. Jt Comm J Qual Patient Saf. 2019;45(1):3-13.

10. Sandbrink F, Uppal R. The time for opioid stewardship is now. Jt Comm J Qual Patient Saf. 2019;45(1):1-2.

11. Opioid stewardship. Institute for Safe Medication Practices Canada. Publié au : https://www.ismp-canada.org/opioid_stewardship/. Consulté le 22 mai 2019.

12. Baldisseri MR. Impaired healthcare professional. Crit Care Med. 2007;35 (2 Suppl):S106-16.

13. Berge KH, Dillon KR, Sikkink KM, Taylor TK, Lanier WL. Diversion of drugs within health care facilities, a multiple-victim crime: patterns of diversion, scope, consequences, detection, and prevention. Mayo Clin Proc. 2012;87(7):674-82.

14. Controlled drugs and substances in hospitals and healthcare facilities: guidelines on secure management and diversion prevention. Canadian Society of Hospital Pharmacists; 2019. Publié au : https://www.cshp.ca/sites/default/files/files/ publications/Official\%20Publications/Guidelines/Controlled\%20Drugs\%20 and $\% 20$ Substances $\% 20$ in $\% 20$ Hospitals $\% 20$ and $\% 20$ Healthcare $\% 20$ Facilities_2019_02-28.pdf. Consulté le 22 mai 2019.

15. Règlement sur les stupéfiants, C.R.C., ch. 1041. Publié au : http://laws-lois. justice.gc.ca/PDF/C.R.C.,_c._1041.pdf. À jour au 20 juin 2018; consulté le 18 juillet 2018.

16. Règlement sur les aliments et drogues, C.R.C., ch. 870. Publié au : http://lawslois.justice.gc.ca/fra/reglements/C.R.C.,_ch._870/ . À jour au 20 juin 2018; consulté le 18 juillet 2018. 
17. Règlement sur les benzodiazépines et autres substances ciblées, DORS/2000-217. Publié au : http://laws.justice.gc.ca/fra/reglements/DORS-2000-217/index. html. À jour au 20 juin 2018; consulté le 18 juillet 2018.

18. Nielsen S, Degenhardt L, Hoban B, Gisev N. A synthesis of oral morphine equivalents (OME) for opioid utilisation studies. Pharmacoepidemiol Drug Saf. 2016;25(6):733-7.

19. Ashton CH. Benzodiazepines : how they work and how to withdraw. Newcastle University, Institute of Neuroscience. Publié au: https://benzo.org.uk/manual/ index.htm. Consulté le 22 mai 2019.

20. Collaborating Centre for Drug Statistics Methodology. ATC/DDD index 2018. Organisation mondiale de la santé. Publié au : https:/www.whocc.no/ atc_ddd_index/. Consulté le 22 mai 2019.

21. Guidelines for ATC classification and DDD assignment 2018. Organisation mondiale de la santé. Publié au : https://www.whocc.no/filearchive/ publications/guidelines.pdf. Consulté le 22 mai 2019.

22. Nielsen S, Gisev N, Bruno R, Hall W, Cohen M, Larance B, et al. Defined daily doses (DDD) do not accurately reflect opioid doses used in contemporary chronic pain treatment. Pharmacoepidemiol Drug Saf. 2017;26(5):587-91.

23. Svendsen K, Borchgrevink P, Fredheim O, Hamunen K, Mellbye A, Dale O. Choosing the unit of measurement counts: the use of oral morphine equivalents in studies of opioid consumption is a useful addition to defined daily doses. Palliat Med. 2011;25(7):725-32.

24. Jarlbaek L, Andersen M, Hallas J, Engholm G, Kragstrup J. Use of opioids in a Danish population-based cohort of cancer patients. J Pain Symptom Manage. 2005;29(4):336-43.

25. Brandt J, Alkabanni W, Alessi-Severini S, Leong C. Translating benzodiazepine utilization data into meaningful population exposure: integration of two metrics for improved reporting. Clin Drug Invest. 2018;38(7):565-572.

26. Islam MM, Conigrave KM, Day CA, Nguyen Y, Haber PS. Twenty-year trends in benzodiazepine dispensing in the Australian population. Intern Med J. 2014;44(1):57-64

27. Nathwani D, Varghese D, Stephens J, Ansari W, Martin S, Charbonneau C. Value of hospital antimicrobial stewardship programs [ASPs]: a systematic review. Antimicrob Resist Infect Control. 2019;8:35.

28. WHO methodology for a global programme on surveillance of antimicrobial consumption. Version 1.0. Organisation mondiale de la santé. Publié au: https://www.who.int/medicines/areas/rational_use/WHO_AMC surveillance_1.0.pdf. Consulté le 22 mai 2019.

29. Canadian Antimicrobial Resistance Surveillance System - update 2018. Publié au : https://www.canada.ca/en/public-health/services/publications/drugshealth-products/canadian-antimicrobial-resistance-surveillance-system-2018report-executive-summary.html. Consulté le 22 mai 2019.

30. Proposition d'un plan de surveillance intégrée de la résistance aux antibiotiques. Institut national de santé publique du Québec; 2017. Publié au : https://www.inspq.qc.ca/sites/default/files/publications/2321_plan_ surveillance_resistance_antibiotiques.pdf. Consulté le 22 mai 2019.

31. Bitterman R, Hussein K, Leibovici L, Carmeli Y, Paul M. Systematic review of antibiotic consumption in acute care hospitals. Clin Microbiol Infect. 2016;22(6):561.e7-561.e19.

32. Anesthésie sans opiacés. Société Française d'anesthesie et de reanimation. Publié au : http://sfar.org/wp-content/uploads/2017/10/Beloeil-Anesthesie-sansopiaces.pdf. Consulté le 22 mai 2019.

33. Rappel et avis de sécurité : Après examen, Santé Canada recommande que la codéine soit administrée seulement chez les patients âgés de 12 ans et plus. Gouvernement du Canada; 6 juin 2013. Publié au : http:// canadiensensante.gc.ca/recall-alert-rappel-avis/hc-sc/2013/33915a-fra.php. Consulté le 22 mai 2019
34. Rappel et avis de sécurité : Nouvelles mesures de sécurité pour resserrer encore l'utilisation de la codéine et de l'hydrocodone sur ordonnance chez les enfants et les adolescents. Gouvernement du Canada; 28 juillet 2016 Publié au : http://canadiensensante.gc.ca/recall-alert-rappel-avis/hc-sc/2016/59584 a-fra.php. Consulté le 22 mai 2019.

35. Santé Canada déconseille l'utilisation de produits contre la toux et le rhume contenant des opioïdes chez les enfants et les adolescents. Gouvernement du Canada; 18 février 2019. Publié au : http://canadiensensante.gc.ca/recall-alertrappel-avis/hc-sc/2019/69080a-fra.php. Consulté le 22 mai 2019.

36. Pan-Canadian trends in the prescribing of opioids, 2012 to 2016. Institut canadien d'information sur la santé. Publié au : https://secure.cihi.ca/ free_products/pan-canadian-trends-opioid-prescribing-2017-en-web.pdf. Consulté le 22 mai 2019.

37. Crise des opioïdes au Canada. Gouvernement du Canada. Publié au : https://www.canada.ca/fr/services/sante/campagnes/prevention-toxicomanie. html. Consulté le 22 mai 2019.

38. Narcotic drugs - technical report. International Narcotics Control Board. Publié au : https://www.incb.org/incb/fr/narcotic-drugs/Technical_Reports/ narcotic_drugs_reports.html. Consulté le 22 mai 2019.

Manon Videau est candidate au Pharm. D. à I'Université Claude Bernard Lyon 1, Lyon, France, et assistante de recherche clinique, Unité de recherche en pratique pharmaceutique, Centre hospitalier universitaire Sainte-Justine, Montréal (Québec).

Maxime Thibault, B. Pharm., M. Sc., est pharmacien responsable des systèmes d'information, Département de pharmacie et Unité de recherche en pratique pharmaceutique, Centre hospitalier universitaire SainteJustine, Montréal (Québec).

Denis Lebel, B. Pharm., M. Sc., FCSHP, est chef-adjoint aux soins pharmaceutiques, enseignement et recherche, Département de pharmacie et Unité de recherche en pratique pharmaceutique, Centre hospitalier universitaire Sainte-Justine, Montréal (Québec).

Suzanne Atkinson, B. Pharm., M. Sc., est chef-adjointe aux services pharmaceutiques, Département de pharmacie et Unité de recherche en pratique pharmaceutique, Centre hospitalier universitaire Sainte-Justine, Montréal (Québec).

Jean-François Bussières, B. Pharm., M. Sc., MBA, FCSHP, FOPQ, est chef de Département de pharmacie et Unité de recherche en pratique pharmaceutique, Centre hospitalier universitaire Sainte-Justine, et professeur titulaire de clinique, Faculté de pharmacie, Université de Montréal, Montréal (Québec).

Conflits d'intérêts: Aucun déclaré.

Adresse de correspondance :

Jean-François Bussières

Département de pharmacie

Centre hospitalier universitaire Sainte-Justine

3175, chemin de la Côte Sainte-Catherine

Montréal QC H3T 1C5

Courriel : jf.bussieres@ssss.gouv.qc.ca

Financement : Aucun reçu. 